\title{
Editorials
}

\section{Can innovation help us deliver an NHS for the 21st century?}

Since its foundation in 1948 the NHS has remained true to its core principles:

1. It meets the needs of everyone;

2. It is free at the point of delivery; and

3. It is based on clinical need and not the ability to pay.

Although almost 70 years have passed since then, our values haven't changed. However, the clinical and social challenges we are presented with have. Our population is living longer, often with multiple chronic comorbidities, so that now approximately $70 \%$ of our healthcare spend is now allocated to the management of chronic disease. Does the system we designed in the 1940s serve us as well now as it did then? In 2017 if we were to design a new NHS would we start from here? How can we utilise the latest, greatest innovations to deliver the healthcare service we need?

\section{HEALTHCARE NEEDS ARE CHANGING GLOBALLY}

The challenges presented by an ageing population with multiple chronic conditions are not unique to the UK or the NHS. Many countries across our planet are grappling with how best to deal with these issues and deliver the often quoted triple aims of better health, better care, delivered with higher quality and at a lower cost. Nations have faced grand challenges in the past and the words of Abraham Lincoln in his speech to Congress in December 1862 ring true now for the challenges we face in health care:

'The dogmas of the quiet past, are inadequate to the stormy present. The occasion is piled high with difficulty, and we must rise with the occasion. As our case is new, so we must think anew, and act anew. We must disenthrall ourselves, and then we shall save our country.

The future of healthcare often appears to be piled high with difficulty. Sometimes being the largest, longest established healthcare system in history can be perceived as a barrier to the type of change necessary to delivering an NHS fit for future generations. However, if there is a place on the planet to innovate in health care at scale, the NHS is it.

\section{A VISION FOR THE FUTURE}

To address these grand healthcare

\section{if there is a place on the planet to innovate in health care at scale, the NHS is it"}

challenges the NHS published its Five Year Forward View (5YFV) in October 2014.' This identified three key areas that needed to be addressed

1. A health and wellbeing gap - to be addressed with a radical upgrade in prevention;

2. A care and quality gap - new models of care will help us deliver a healthcare system with features complementary to 'accountable care' type systems; and

3. A funding gap - a quality, efficiency, and investment programme is starting to address this.

Innovation is at the heart of delivering this transformative vision. It acknowledges that we not only have to 'do things better' but also 'do better things'

In addition to the changes set out in the 5YFV, we also have to deliver on the personalised, empowered health and care revolution that is upon us. The latest developments in omics, advanced technology, data analytics, machine learning, artificial intelligence, digital health, and social networking will help to make this revolution a reality in the next 5-10 years. Increasingly, solutions in these key areas are being invented and delivered by industry, for example, companies like DeepMind are harnessing the power of machine learning in partnership with the NHS to report Optical Coherence Tomography scans of the retina and assist in radiotherapy planning in oral cancer (https://deepmind. com/applied/deepmind-health/research/). The NHS needs the way it provides care to be transformed, so we need to develop new relationships between industry and professionals, the pathway from research to adopted innovation needs to be unified, and a strategy for economic growth in the health sector delivered.

These changes are heralding the most disruptive era ever seen in medicine, with more and more patients taking control of their health care. The medical profession is likely to have their role as the gatekeepers of health care increasingly replaced with the position of system navigator and advisor.

This combination of medical grand challenges and technological advances are setting the pace. If we are to deliver a healthcare system that is both true to the founding principles of the NHS and fit for the future, we have to innovate and do it at scale, but how can that be achieved? Furthermore, how can proven innovations be adopted into best practice and taken up across the whole healthcare system rapidly?

\section{HOW IS THE NHS GOING TO DELIVER ON INNOVATION?}

In a word, the answer is culture. If we get that right, the transformation we need will follow. Many of the building blocks that will help form the foundation of a healthcare innovation culture are already in place. ${ }^{2}$ Some of the key culture-building components are listed in Box 1.

\section{CLINICAL ENTREPRENEURS}

Many of the latest, greatest advances that are changing the way we live our lives are arising from industry and in particular from the start-up sector. Business models that once served companies well are being disrupted and increasingly this is coming from outside their sector. For example, Airbnb probably provides more overnight accommodation than many of the global hotel chains added together, but doesn't own any hotels, Amazon is one of the biggest retailers on the planet but doesn't have any high street shops, and Uber is one of the largest providers of 'taxi rides' but doesn't own any taxis. Only time will tell who will be the disruptors of health care. In the UK, we have not traditionally been as supportive of entrepreneurial clinicians as some other countries. If we are to deliver on the promise of the 5YFV and the personalised, empowered healthcare revolution we need to equip our clinicians with the entrepreneurial skills, knowledge, and experience that will enable them to understand and engage with this new 


\section{clinical entrepreneurs will ultimately number in the thousands and will act as frontline agents for change, adoption and spread of innovation}

\section{Box 1. Key NHS initiatives that contribute to a culture supporting innovation}

- Best Practice Tariffs: incentivise and reimburse providers for the cost of highquality, innovative care.

- Innovation Challenge Prizes: a national-level celebration and reward for outstanding innovation in health care.

- Healthcare Small Business Research Initiative: pre-commercial procurement contracts for research and development in innovative healthcare products and services.

- Clinical Excellence Awards: rewarding clinicians for quality improvement, research and innovation.

- Academic Health Science Networks: 15 regional organisations bringing together health care, academia, and industry on an open innovation platform to lead work in the NHS on adoption and diffusion of innovation.

- NHS England Innovation portal: Innovation Connect and Innovation Exchange provides support, advice, and navigation through the system and is open to all who want to innovate and collaborate in health care.

- Innovation Scorecard: enables benchmarking and increases transparency for the variation of adoption in NICE Technology Appraisals.

- Test Beds: real-world sites testing combinatorial innovations that can deliver better care and better patient experience of care at the same or lower overall cost.

- National Innovation Accelerator: a fellowship programme equipping innovators with the skills, knowledge, and experience to drive the uptake of their proven innovations across the NHS.

- Health and Social Care Innovation Expo (https://www.england.nhs.uk/expo/).

- Healthy New Towns programme: improving health through the built environment, reshaping the health of communities, and rethinking how health and care services can be delivered.

- A Continuing Clinical Innovation (CCI) points scheme: a framework will be provided that allows clinicians and their appraisers to award $\mathrm{CCl}$ points for innovative activity that will count towards their annual appraisal and revalidation.

- The Innovation Technology Tariff: nationallevel reimbursement for new medtech innovations.

- The Clinical Entrepreneur Programme: a workforce development programme allowing clinicians to continue their frontline NHS roles while developing their entrepreneurial ideas. world. We need to develop our clinicians so that they have both entrepreneurial and intrapreneurial abilities.

To address this, NHS England has launched the Clinical Entrepreneur Programme (https://www.innovation england.nhs.uk/clinical-entrepreneur). This is a national-scale workforce development initiative that allows clinicians in training to undertake entrepreneurial activity alongside their clinical work. It provides a coaching and mentoring scheme, less than full-time training opportunities, advanced industry internships, customer matching education, and networking events. In its first year, 103 of the country's brightest, most entrepreneurial clinicians were appointed. One-third of them were from primary care. In future years, the programme will expand to include all clinical professionals, patients, and citizens. By including all as we redesign health care, we will have the best chance of getting it right.

The clinical entrepreneurs will ultimately number in the thousands and will act as frontline agents for change, adoption and spread of innovation throughout the NHS and beyond.

\section{INNOVATION NEVER STOPS}

In spite of the sizeable innovation effort we are currently undertaking, we cannot rest. We still need to do more. We need to accelerate the process from idea to innovation spread throughout the system and join up the pockets of excellence in innovation, gaining system-wide adoption. Creating a vision and strategy for economic growth within the health sector and making the NHS a straightforward place to do business will help drive this.

The most valuable asset the NHS has is its workforce. We should provide our innovative leaders of the future with new opportunities in the training and education and clinicial entrepreneur training posts.

\section{CONCLUSION}

The healthcare issues facing the NHS in the 21st century are of a different nature and scale from anything we have seen before. Adopting and diffusing innovation system by supporting more clinical leader

\section{ADDRESS FOR CORRESPONDENCE}

\section{Tony Young}

Anglia Ruskin University, Postgraduate Medical Institute, Chelmsford Campus, Bishop Hall Lane, Chelmsford, CM1 1SQ, UK

\section{E-mail: tony.youngdanglia.ac.uk}

across the system is no longer an option; it must form the foundation of a strategy to meet the demands we now face. We need to de-innovate the things that once served us well and utilise the advances in omics, advanced technology, data analytics, machine learning, digital health, and social networking. However, most importantly we need to empower our workforce and support them with their innovative ideas. The challenges we face are soluble, but to continue delivering a high-quality healthcare system, free at the point of delivery land to paraphrase Theodore Roosevelt's 'Man in the Arena' speech) - we all need to roll up our sleeves, get out of our seats, and enter the healthcare arena.

\section{Tony Young,}

Professor, National Clinical Lead for Innovation NHS England, Director of Medical Innovation Anglia Ruskin University, Consultant Urological Surgeon Southend University Hospital.

\section{Provenance}

Freely submitted; not externally peer reviewed.

DOI: https://doi.org/10.3399/bjgp17X690053

\section{REFERENCES}

1. NHS. Five year forward view. 2014 https://www.england.nhs.uk/wp-content/ uploads/2014/10/5yfv-web.pdf laccessed 7 Mar 2017).

2. NHS. Innovation into action. Supporting delivery of the NHS five year forward view. 2015. https://www.england.nhs.uk/ wp-content/uploads/2015/10/nhs-inovationinto-action.pdf (accessed 7 Mar 2017). 